\title{
Long non-coding RNA XIST predicts poor prognosis and promotes malignant phenotypes in osteosarcoma
}

\author{
WEI WANG, HUILIANG SHEN, GUANGLEI CAO and JIANG HUANG \\ Department of Orthopedics, Xuanwu Hospital, Capital Medical University, Beijing 100053, P.R. China
}

Received October 31, 2017; Accepted August 9, 2018

DOI: $10.3892 / \mathrm{ol} .2018 .9596$

\begin{abstract}
Increasing evidence indicates that long non-coding RNA (lncRNA) is involved in a number of types of human cancer and functions as an oncogene or tumor suppressor. However, little is known about the role of lncRNA X-inactive specific transcript (XIST) in osteosarcoma. In the present study, the expression of IncRNA XIST was analyzed in 56 osteosarcoma tissues and their corresponding normal bone tissues by reverse transcription-quantitative polymerase chain reaction. Increased lncRNA XIST expression was observed in osteosarcoma tissues compared with the normal tissues and was positively associated with advanced Enneking stage. In addition, lncRNA XIST may be used as an independent risk factor for the overall survival of patients with osteosarcoma. Knockdown of lncRNA XIST inhibited cell proliferation, induced cell apoptosis and arrested the cell cycle. The inhibition of 1ncRNA XIST may also suppress cell migration and invasion in vitro. The present study suggested that lncRNA XIST acted as an oncogene in osteosarcoma, which may make it a novel therapeutic target for the treatment of osteosarcoma.
\end{abstract}

\section{Introduction}

Osteosarcoma (OS) is one of the most frequently diagnosed malignancies in child and adolescent populations $(1,2)$. With the advance of multiple therapeutic strategies, including surgical resection, adjuvant chemotherapy and radiotherapy, the survival rate of OS patients has significantly increased $(3,4)$. However, the 5-year survival rate remains poor due to local relapse and metastasis, especially pulmonary metastasis, after resection of the primary OS $(4,5)$. Therefore, research is needed to further elucidate the complex molecular mechanisms underlying OS tumorigenesis and progression and to identify novel early molecular markers of diagnostic and therapeutic targets for OS.

Correspondence to: Dr Jiang Huang, Department of Orthopedics, Xuanwu Hospital, Capital Medical University, 45 Changchun Street, Xicheng, Beijing 100053, P.R. China

E-mail: xxch002hj@163.com

Key words: osteosarcoma, long non-coding RNA, XIST, prognosis
Long non-coding RNAs (lncRNAs) are more than 200 nucleotides in length, endogenously expressed, and have important biological functions (6-8). Increasing evidence indicates that lncRNAs play critical roles in the modulation of cellular biological function, such as differentiation, apoptosis and metastasis (9-12). Several specific lncRNAs have been reported to increase in OS and serve as a diagnostic marker for OS (13-17). The lncRNA X-inactive specific transcript (XIST), derived from XIST gene, was found to dysregulate in many cancers and play an important role in cell proliferation, differentiation and genome maintenance (18-22). For example, lncRNA XIST was increased in aggressive tumor phenotypes and down-regulated the expression of EZH2 via miR-101 in gastric cancer (18). IncRNA XIST was found to be over-expressed in non-small-cell lung carcinoma (NSCLC) and this high level was associated with a shorter survival rate and poorer prognosis. Knockdown of XIST impaired NSCLC cell proliferation, migration and invasion by interacting with EZH2 to suppress the transcription of KLF2 (19). More recently, it has been shown that upregulation of XIST contributes to cell proliferation and invasion by inhibiting miR-497 expression (23). However, biological roles and underlying molecular mechanisms of XIST in OS tumorigenesis remain largely unknown.

In our study, we explored the expression and roles of XIST in OS progression. We found the expression level of XIST was increased in OS tumor tissues, and high XIST expression was associated with Enneking stage, metastasis and a short overall survival rate. Furthermore, knockdown of XIST inhibited cell proliferation, migration and invasion.

\section{Materials and methods}

Tissue specimens. A total of 64 pairs of OS tissues and their matched adjacent normal bone were obtained from patients who underwent surgery at The Xuanwu Hospital of Capital Medical University (Beijing, China) from March 2008 to October 2009. All patients' operation treatments were performed by the same senior doctors according to the patients' Enneking staging. None of the patients had received radiotherapy or chemotherapy before surgery. This study was approved by the Research Ethics Committee of the Capital Medical University with the permit no. 2014A083. Written informed consent was acquired from all the patients. Tissue specimens were immediately frozen in liquid nitrogen after surgery and stored at $-80^{\circ} \mathrm{C}$ until use. 
Cell culture. OS cell lines (KHOS-240S, SaOS2, MG-63, SOSP-9607 and U2OS), and osteoblastic cell line (hFOB1.19) were purchased from the Cell Bank of Type Culture Collection of Chinese Academy of Sciences (Shanghai, China). All cells were cultured in Dulbecco's modified Eagle's medium or Ham's F12/Dulbecco's modified Eagle's medium (both GE Healthcare, Logan, UT, USA) supplemented with $10 \%$ fetal bovine serum (Gibco; Thermo Fisher Scientific, Inc., Waltham, MA, USA), $100 \mathrm{U} / \mathrm{ml}$ of penicillin and $100 \mathrm{mg} / \mathrm{ml}$ of streptomycin at $37^{\circ} \mathrm{C}$ in a humidified atmosphere with $5 \% \mathrm{CO}_{2}$.

RNA isolation and quantitative real-time PCR (qRT-PCR). Total RNA was extracted from the tissue samples and cells using TRIzol reagent (Invitrogen; Thermo Fisher Scientific, Inc.) according to the manufacturer's instructions. The first-strand cDNA was reverse-transcribed from $500 \mathrm{ng}$ of total RNA using a PrimeScript ${ }^{\mathrm{TM}}$ II 1st Strand cDNA Synthesis kit (Takara, Dalian, China). qRT-PCR was performed using the FastStart Universal SYBR-Green Master Mixes (Roche Diagnostics Corp., Indianapolis, IN, USA) on a 7900 Fast Real-Time PCR system (Applied Biosystems, Foster City, CA, USA). The following primers were used to detect the expression of XIST and GAPDH: XIST sense, 5'-CTCTCCATT GGGTTCAC-3' and reverse, 5'-GCGGCAGGTCTTAAGAGA TGAG-3'; GAPDH sense, 5'-AGAAGGCTGGGGCTCATT TG-3' and reverse, 5'-AGGGGCCATCCACAGTCTTC-3'. The expression of XIST was normalized to GAPDH.

Cell transfection. The small interfering RNA (siRNA) specifically targeting XIST was designed and commercially constructed by GenePharma (Shanghai, China). The scrambled nucleotide was used as the negative control (si-NC). The sequences were as follows: siRNA1 sense, 5'-GUAUCC UAUUUGCACGCUAdTdT-3'; siRNA2 sense, 5'-GCCCUU CUCUUCGAACUGUdTdT-3'; negative control siRNA sense, 5'-UUCUCCGAACGUGUCACGUdTdT-3'. Cells were transfected separately with the siRNAs and si-NC using FuGENE 6 transfection reagent (Roche Diagnostics) to knockdown the XIST. The interfering efficiency was confirmed by qRT-PCR after transfection for $48 \mathrm{~h}$.

Cell proliferation assay. Cell proliferation assay was used to examine the effect of XIST knockdown on viability of OS cells. In brief, $2 \times 10^{3}$ cells transfected with MG-63 and U2OS were seeded into 96-well plates. At 24, 48, 72, and $96 \mathrm{~h}, 10 \mu \mathrm{l}$ Cell Counting kit-8 (CCK-8) solution (Dojindo, Tokyo, Japan) was added into each well and incubated for $2 \mathrm{~h}$ at $37^{\circ} \mathrm{C}$. The absorbance was measured on a microplate reader at $450 \mathrm{~nm}$ by Biotek Elx800 ELISA (BioTek, Winooski, VT, USA).

Flow cytometry analysis. For cell apoptosis analysis, cells were harvested and stained with FITC-Annexin V and propidium iodide (PI; BD Biosciences, San Jose, CA, USA). The fluorescence of stained cells was then examined using flow cytometry (BD Biosciences) according to the manufacturer's protocol. For cell cycle analysis, cells were harvested, washed with cold PBS, and fixed in $70 \%$ ethanol at $4^{\circ} \mathrm{C}$ overnight. The cells were then stained with PI in the dark for $15 \mathrm{~min}$ at room temperature. The proportion of cells in the $\mathrm{G} 0 / \mathrm{G} 1, \mathrm{~S}$, and G2/M phases were determined by flow cytometry.
Table I. Correlation between XIST and clinicopathologic factors in patients with osteosarcoma.

\begin{tabular}{|c|c|c|c|}
\hline \multirow[b]{2}{*}{ Variables } & \multicolumn{2}{|c|}{ XIST expression } & \multirow[b]{2}{*}{ P-value } \\
\hline & Low $(n=32)$ & High $(n=32)$ & \\
\hline Sex & & & 0.3171 \\
\hline Female & 14 & 19 & \\
\hline Male & 18 & 13 & \\
\hline Age, years & & & 0.5950 \\
\hline$\leq 18$ & 20 & 23 & \\
\hline$>18$ & 12 & 9 & \\
\hline Enneking stage & & & 0.0051 \\
\hline IA-IIA & 24 & 12 & \\
\hline IIB-III & 8 & 20 & \\
\hline Tumor size, $\mathrm{cm}$ & & & 0.3114 \\
\hline$<5$ & 16 & 21 & \\
\hline$\geq 5$ & 16 & 11 & \\
\hline Pathologic fracture & & & 0.7323 \\
\hline Absent & 28 & 26 & \\
\hline Present & 4 & 6 & \\
\hline Location & & & 0.7818 \\
\hline Upper limbs & 7 & 8 & \\
\hline Lower limbs & 19 & 20 & \\
\hline Other & 6 & 4 & \\
\hline Metastasis & & & 0.0120 \\
\hline Absent & 29 & 22 & \\
\hline Present & 3 & 12 & \\
\hline
\end{tabular}

XIST, X-inactive specific transcript.

Cell migration and invasion assays. Cell migration ability was assessed using a 24 -well plate with $8-\mu \mathrm{m}$ pore size chamber inserts (Corning Inc., New York, NY, USA). Cell invasion ability was assessed using a 24 -well plate with $8-\mu \mathrm{m}$ pore size chamber inserts coated with Matrigel (BD Biosciences). $5 \times 10^{4}$ cells were suspended in $150 \mu \mathrm{l}$ of serum-free medium and were placed in the upper chamber. Six hundred $\mu 1$ of medium containing $10 \%$ FBS was added to the lower chamber as the chemoattractant. Following incubation for $36 \mathrm{~h}$ at $37^{\circ} \mathrm{C}$, the non-invading cells were gently removed with a cotton swab. Invasive cells located on the lower side of the chamber were fixed in $95 \%$ ethanol and stained with $0.5 \%$ crystal violet. The numbers of cells in five randomly selected fields were counted, and the cells were imaged through a CKX41 inverted microscope (Olympus Corp., Tokyo, Japan).

Western blot analysis. Total protein from cells was extracted by lysing cells in RIPA buffer (Sigma-Aldrich; Merck KGaA, Darmstadt, Germany) containing protease and phosphatase inhibitors (Roche Diagnostics). Equivalent protein lysates were separated by $10 \%$ sodium dodecyl sulfate-polyacrylamide gel electrophoresis and transferred to polyvinylidene fluoride membranes (EMD Millipore, Billerica, MA, USA). The membranes were then blocked using blocking buffer 

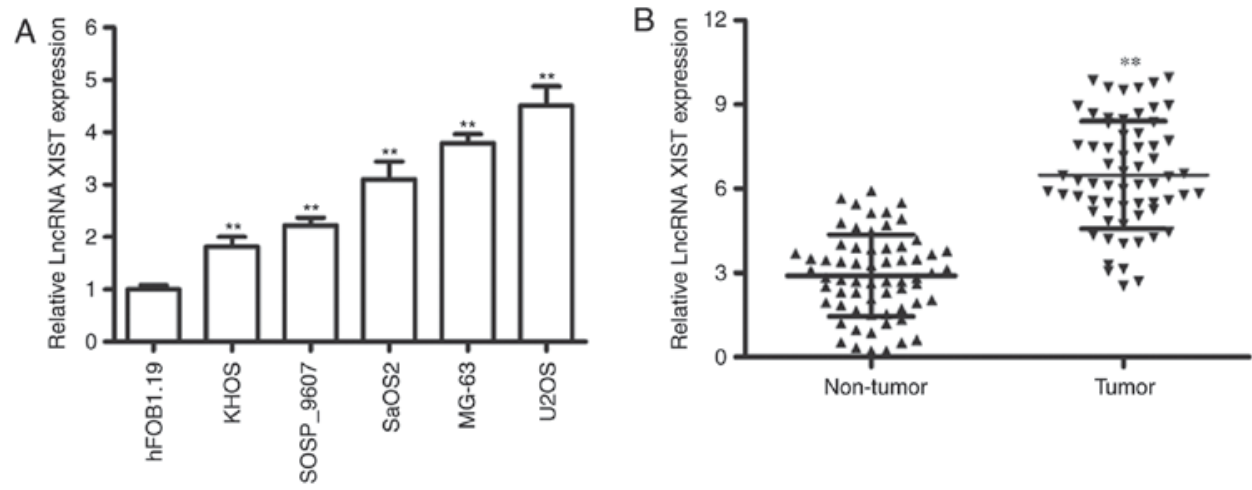

Figure 1. Expression of IncRNA XIST in osteosarcoma cell lines and clinical tissues. (A) Relative expression of XIST in four osteosarcoma cell lines and normal osteoblast cells. (B) The expression of XIST was significantly higher in osteosarcoma samples compared with adjacent non-tumor tissues. ${ }^{* *} \mathrm{P}<0.01$ vs. the control. LncRNA, long non coding RNA; XIST, X-inactive specific transcript.

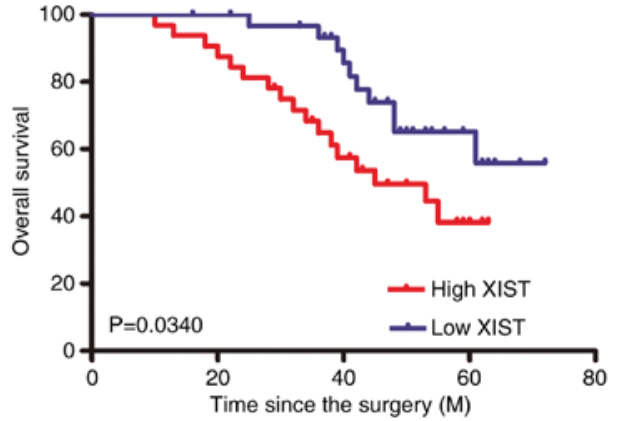

Figure 2. Overall survival of 64 patients with osteosarcoma in association with XIST expression. Kaplan-Meier survival curves revealed that patients in the high XIST expression group had significantly shorter overall survival rates compared with those in the low XIST expression group. XIST, $\mathrm{X}$-inactive specific transcript.

and then incubated with primary antibody overnight at $4{ }^{\circ} \mathrm{C}$ After the membranes were incubated with secondary antibody, the signals were visualized using a SuperSignal West Pico Chemiluminescent Substrate kit (Pierce; Thermo Fisher Scientific, Inc.). Antibodies against Vimentin, c-Myc, E-cadherin, and GAPDH were purchased from Cell Signaling Technology (Danvers, MA, USA).

Statistical analysis. All the experiments were repeated in triplicate, and data was expressed as the mean \pm standard deviation (SD). Statistical analyses were performed using SPSS 18.0 software package (SPSS Inc, Chicago, IL, USA). One-way or two-way analyses of variance was performed with Dunnett's post hoc test to determine statistical differences between $>2$ groups. The Chi-squared test was used to evaluate the association between the XIST expression and the clinicopathological features. The Kaplan-Meier method and log-rank test were used for the survival analysis. $\mathrm{P}<0.05$ was considered to indicate a statistically significant difference.

\section{Results}

LncRNA XIST is highly expressed in OS cell lines and tissues. To evaluate the expression of XIST, we measured the expression of XIST in OS cell lines and our results indicated that the expression level of XIST was relatively high in four OS cell lines, including KHOS-240S, SaOS2, MG-63 and U2OS, compared to the osteoblastic cell line hFOB1.19 (Fig. 1A). Furthermore, we determined the levels of XIST expression in 64 pairs of OS tissues and adjacent non-tumor tissues. The results suggested that XIST was significantly increased in the cancerous tissues compared with the adjacent normal samples (Fig. 1B). Our results suggested that significant upregulation of XIST was frequently observed in the majority of the OS tumors and cell lines.

High levels of IncRNA XIST predict poor prognosis in OS patients. To assess the clinical significance, OS tissues were divided into two groups including the high XIST expression group $(n=32)$ (higher than median value) and the low XIST expression group $(\mathrm{n}=32)$ (lower than median value) according to the median value of XIST expression level of all samples. As shown in Table I, the high XIST expression group was positively associated with Enneking stage and metastasis. However, there was no significant correlation between XIST expression and other clinicopathological features such as sex, age, tumor location, tumor size and pathologic fracture. In addition, Kaplan-Meier survival curves showed that patients in the high XIST expression group had a worse overall survival rate than those in the low XIST expression group (Fig. 2).

Knockdown of LncRNA XIST reduced HCC cell growth. Since XIST was significantly upregulated in OS tissues, we investigated the biological function of XIST silencing in OS cells. XIST was downregulated by transfecting the siRNAs against XIST into the MG-63 and U2OS cell lines, which harbored the highest expression level of XIST (Fig. 3A). Knockdown of XIST markedly impaired the growth of MG-63 and U2OS cells compared to the NC-transfected cells (Fig. 3B). The siRNA\#1 against XIST had more efficient inhibition and was chosen for the following experiments. Furthermore, compared to si-NC, transfection with XISTsiRNA\#1 resulted in a considerable increase of the apoptotic percentage in MG-63 and U2OS cells (Fig. 3C). Additionally, XIST silencing exhibited a significant decrease in the percentage of cells in $\mathrm{S}$ phase and an increase in cells in the $\mathrm{G} 2 / \mathrm{M}$ phase compared with si-NC in both MG-63 and U2OS cells (Fig. 3D). These data suggested that XIST promoted cell proliferation by mediating cell apoptosis and cell cycle in vitro. 

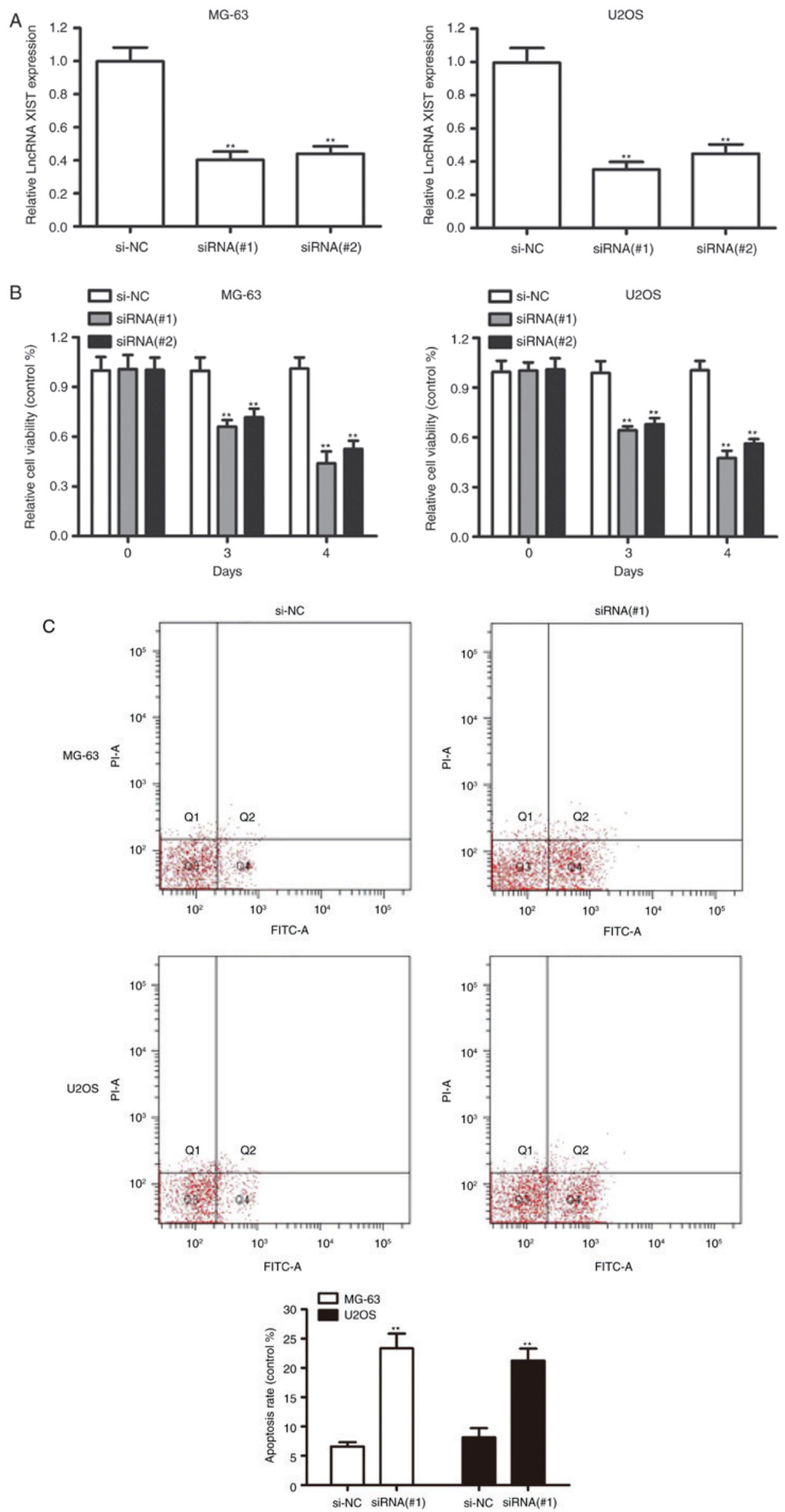

Figure 3. Effect of XIST on gastric cancer cell proliferation and cell cycle. (A) The expression of XIST in MG-63 and U2OS transfected with si-NC or siRNA against XIST was confirmed by reverse transcription-quantitative polymerase chain reaction. (B) Cell counting kit-8 assays, (C) Annexin V/phycoerythrin staining and flow cytometry analysis was used to determine the effects of XIST silencing on OS cells. ${ }^{* *} \mathrm{P}<0.01$. 

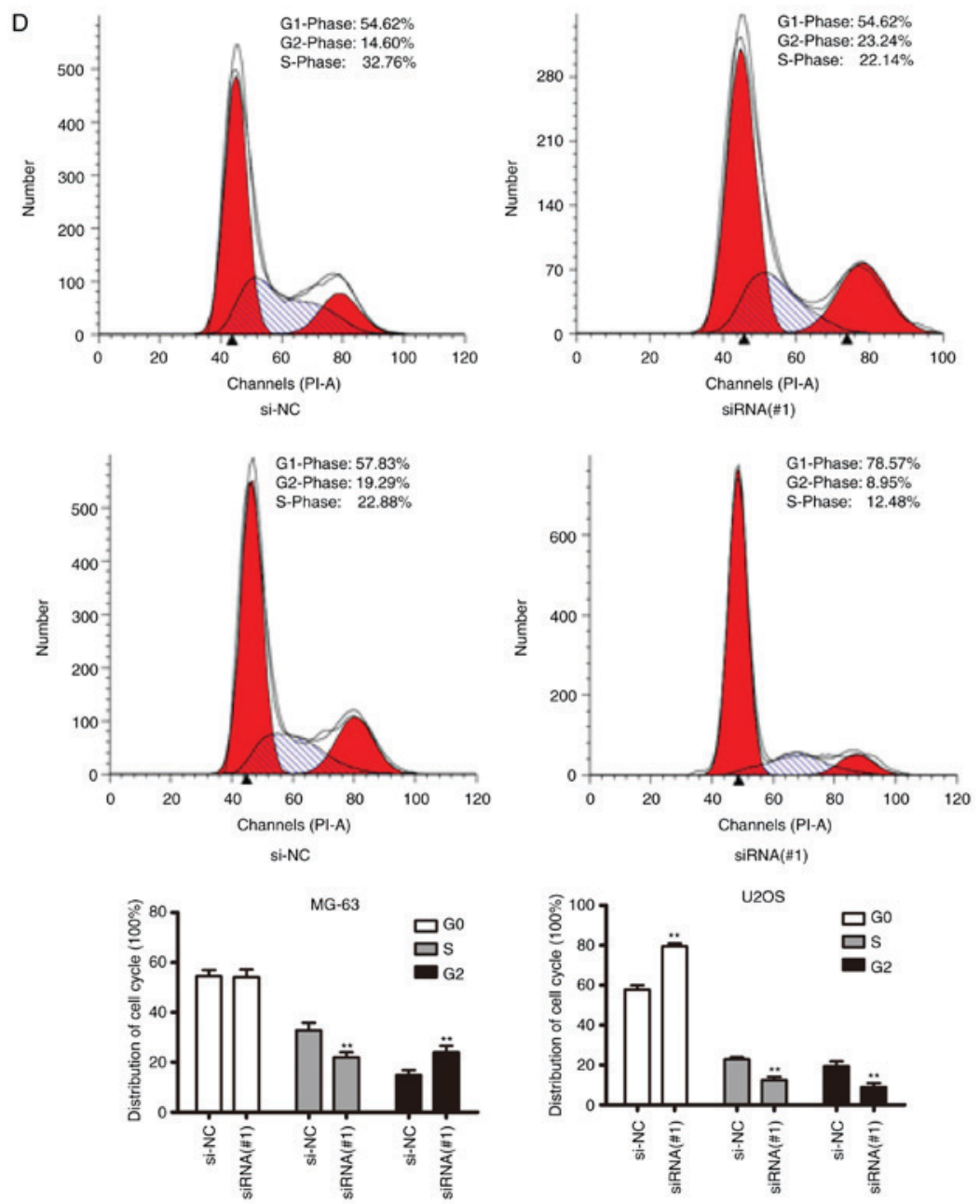

Figure 3. Continued. (D) cell cycle analysis was used to determine the effects of XIST silencing on OS cells. ${ }^{* *} \mathrm{P}<0.01$ vs. the NC group. OS, osteosarcoma; si, short interfering; $\mathrm{NC}$, negative control.

Inhibition of LncRNA XIST impeded cell migration and invasion. To investigate the potential role of XIST in OS metastasis, the present study detected the effect of XIST on the migration and invasion capacity of OS cells. We found that XIST knockdown dramatically decreased their migration and invasion capabilities in both MG-63 (Fig. 4A) and U2OS (Fig. 4B) cells. We then sought to explore whether there was some interaction between proliferation- and metastasis-related markers and XIST expression. Interestingly, XIST knockdown upregulated E-cadherin and repressed Vimentin and c-Myc (Fig. 4C), which are clearly involved in OS cell proliferation and invasion. These results indicated that XIST functioned as an oncogene and promoted the invasion of OS cells.

\section{Discussion}

In our present study, we found that XIST was expressed significantly higher in OS cell lines and tissues. Further investigation indicated that high XIST expression was positively associated with advanced Enneking stage and metastasis, suggesting that XIST might contribute to the progression of OS by acting as an oncogene. Our results of the expression of XIST were consistent with previous research demonstrating high XIST expression in other cancers, such as NSCLC, gastric cancer, nasopharyngeal carcinoma and OS $(18-20,23,24)$. In addition, Kaplan-Meier analysis showed that patients with high XIST expression have poorer prognosis in OS. Consistent with our results, previous research has shown that a high expression of XIST was associated with short overall survival rates in patients with gastric cancer and OS $(18,24)$. However, XIST was found to be significantly downregulated in both OS tissues and cell lines, and overexpression of XIST inhibited cell proliferation and invasion in vitro as well as tumor growth in vivo (25). These results suggested that XIST might be involved in the development and progression of OS.

Several studies have emphasized the role of XIST in tumorigenesis and progression in NSCLC and gastric cancer $(18,19,23)$. Knockdown of XIST repressed cell proliferation and tumorigenicity in vitro and in vivo by suppressing KLF2 expression in NSCLC (19). Knockdown of XIST inhibited gastric cancer cell proliferation, migration invasion, and tumor growth by suppressing miR-101, which increased EZH2 expression XIST (18). Deletion of XIST inhibited cell proliferation and invasion through the miR-320b/RAP2B axis (26). Consistent with previous studies, our study showed that XIST silencing significantly inhibited OS cell growth, cell migration and invasion ability, 

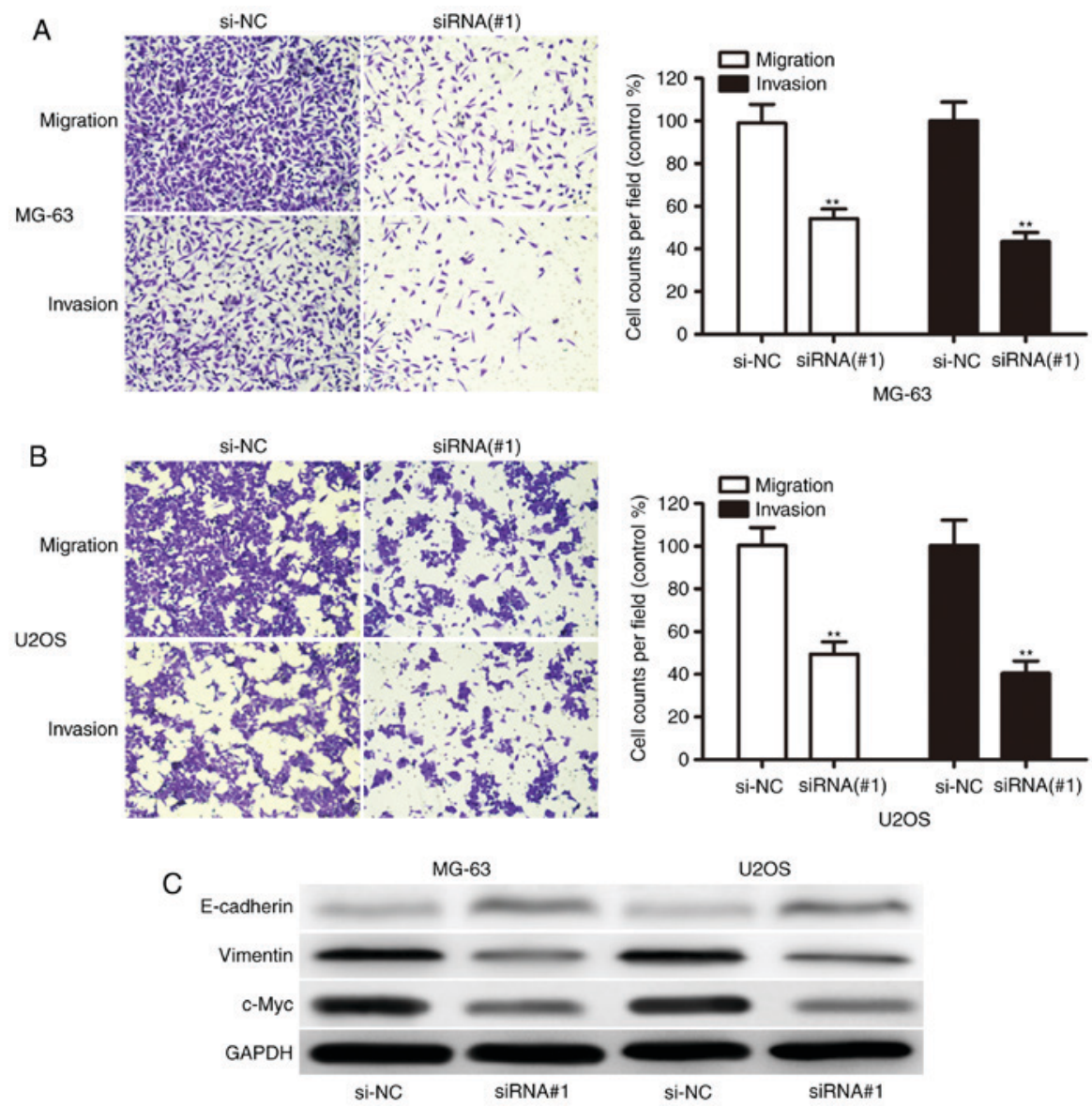

Figure 4. Knockdown of lncRNA XIST inhibited osteosarcoma cell line migration and invasion in vitro. (A) Transwell assays were performed to determine the effect of knockdown of XIST on migration and invasion of (A) MG-63 and (B) U2OS. Original magnification, x100. (C) The protein expression of c-Myc, Vimentin and E-cadherin were altered as a result of XIST knockdown. ${ }^{* *} \mathrm{P}<0.01$ vs. the NC group. si, short interfering; NC, negative control; XIST, X-inactive specific transcript.

as well as inducing cell apoptosis and cell cycle alteration, implying that XIST played an important role in OS progression. C-Myc, Vimentin and E-cadherin have been shown to play critical roles in the processes of tumor cell proliferation and invasion in OS (27-29). Furthermore, we found that the knockdown of XIST resulted in decreased expression of c-Myc and Vimentin and increased E-cadherin expression in OS cells. These results indicated that XIST promoted cell proliferation and invasion by mediating c-Myc, Vimentin and E-cadherin.

In summary, we have provided evidence demonstrating that upregulated XIST expression in OS was significantly associated with advanced Enneking stage and poor prognosis. Knockdown of lncRNA XIST inhibited cell proliferation, migration and invasion in vitro. These findings shed a light on the potential role of XIST in OS pathogenesis and provided a valuable therapeutic target for OS.

\section{Acknowledgements}

Not applicable.

\section{Funding}

The present study was supported by National Natural Science Foundation of China (grant no. 81541135).

\section{Availability of data and materials}

All data generated or analyzed during this study are included in this published article.

\section{Authors' contributions}

WW and JH conceived and designed the study. WW, HS and CG performed the experiments, coordinated the study and wrote the manuscript. All authors read and approved the final manuscript.

\section{Ethics approval and consent to participate}

The present study was approved by the Research Ethics Committee of the Capital Medical University (approval no. 2014A083). Written informed consent was acquired from all patients prior to their inclusion in the study.

\section{Patient consent for publication}

Not applicable.

\section{Competing interests}

The authors declare that they have no competing interests. 


\section{References}

1. Ottaviani G and Jaffe N: The epidemiology of osteosarcoma. Cancer Treat Res 152: 3-13, 2009.

2. Berner K, Johannesen TB and Bruland OS: Clinical epidemiology of low-grade and dedifferentiated osteosarcoma in Norway during 1975 and 2009. Sarcoma 2015: 917679, 2015.

3. Allison DC, Carney SC, Ahlmann ER, Hendifar A, Chawla S, Fedenko A, Angeles C and Menendez LR: A meta-analysis of osteosarcoma outcomes in the modern medical era Sarcoma 2012: 704872, 2012.

4. Ritter J and Bielack SS: Osteosarcoma. Ann Oncol 21 (Suppl 7): vii320-vii325, 2010

5. Yang J and Zhang W: New molecular insights into osteosarcoma targeted therapy. Curr Opin Oncol 25: 398-406, 2013.

6. Ellis BC, Molloy PL and Graham LD: CRNDE: A long non-coding RNA involved in canceR, neurobiology, and DEvelopment. Front Genet 3: 270, 2012.

7. Ponting CP, Oliver PL and Reik W: Evolution and functions of long noncoding RNAs. Cell 136: 629-641, 2009.

8. Kung JT, Colognori D and Lee JT: Long noncoding RNAs: Past, present, and future. Genetics 193: 651-669, 2013

9. Wu CH, Hsu CL, Lu PC, Lin WC, Juan HF and Huang HC: Identification of lncRNA functions in lung cancer based on associated protein-protein interaction modules. Sci Rep 6: 35939, 2016.

10. Niu J, Lin Y, Liu P, Yu Y, Su C and Wang X: Microarray analysis on the lncRNA expression profile in male hepatocelluar carcinoma patients with chronic hepatitis B virus infection. Oncotarget 7: 76169-76180, 2016.

11. Luo W, He H, Xiao W, Liu Q, Deng Z, Lu Y, Wang Q, Zheng Q and Li Y: MALAT1 promotes osteosarcoma development by targeting TGFA via MIR376A. Oncotarget 7: 54733-54743, 2016

12. Qi D, Li J, Que B, Su J, Li M, Zhang C, Yang M, Zhou G and Ji W: Long non-coding RNA DBCCR1-003 regulate the expression of DBCCR1 via DNMT1 in bladder cancer. Cancer Cell Int 16: 81, 2016.

13. Zhou Q, Chen F, Fei Z, Zhao J, Liang Y, Pan W, Liu X and Zheng D: Genetic variants of lncRNA HOTAIR contribute to the risk of osteosarcoma. Oncotarget 7: 19928-19934, 2016.

14. Ma B, Li M, Zhang L, Huang M, Lei JB, Fu GH, Liu CX, Lai QW, Chen QQ and Wang YL: Upregulation of long non-coding RNA TUG1 correlates with poor prognosis and disease status in osteosarcoma. Tumour Biol 37: 4445-4455, 2016.

15. Li Z, Zhao L and Wang Q: Overexpression of long non-coding RNA HOTTIP increases chemoresistance of osteosarcoma cel by activating the Wnt/ $\beta$-catenin pathway. Am J Transl Res 8: 2385-2393, 2016

16. Xia WK, Lin QF, Shen D, Liu ZL, Su J and Mao WD: Clinical implication of long noncoding RNA $91 \mathrm{H}$ expression profile in osteosarcoma patients. Onco Targets Ther 9: 4645-4652, 2016.

17. Wei X, Wang C, Ma C, Sun W, Li H and Cai Z: Long noncoding RNA ANRIL is activated by hypoxia-inducible factor-1 $\alpha$ and promotes osteosarcoma cell invasion and suppresses cell apoptosis upon hypoxia. Cancer Cell Int 16: 73, 2016.
18. Chen DL, Ju HQ, Lu YX, Chen LZ, Zeng ZL, Zhang DS, Luo HY, Wang F, Qiu MZ, Wang DS, et al: Long non-coding RNA XIST regulates gastric cancer progression by acting as a molecular sponge of miR-101 to modulate EZH2 expression. J Exp Clin Cancer Res 35: 142, 2016.

19. Fang J, Sun CC and Gong C: Long noncoding RNA XIST acts as an oncogene in non-small cell lung cancer by epigenetically repressing KLF2 expression. Biochem Biophys Res Commun 478: 811-817, 2016.

20. Song P, Ye LF, Zhang C, Peng T and Zhou XH: Long non-coding RNA XIST exerts oncogenic functions in human nasopharyngeal carcinoma by targeting miR-34a-5p. Gene 592: 8-14, 2016.

21. Schouten PC, Vollebergh MA, Opdam M, Jonkers M, Loden M, Wesseling J, Hauptmann M and Linn SC: High XIST and Low 53BP1 expression predict poor outcome after high-dose alkylating chemotherapy in patients with a BRCA1-like breast cancer. Mol Cancer Ther 15: 190-198, 2016.

22. Huang YS, Chang CC, Lee SS, Jou YS and Shih HM: Xist reduction in breast cancer upregulates AKT phosphorylation via HDAC3-mediated repression of PHLPP1 expression. Oncotarget 7: 43256-43266, 2016.

23. Ma L, Zhou Y, Luo X, Gao H, Deng X and Jiang Y: Long non-coding RNA XIST promotes cell growth and invasion through regulating miR-497/MACC1 axis in gastric cancer. Oncotarget 8: 4125-4135, 2017.

24. Li GL, Wu YX, Li YM and Li J: High expression of long non-coding RNA XIST in osteosarcoma is associated with cell proliferation and poor prognosis. Eur Rev Med Pharmacol Sci 21: 2829-2834, 2017

25. Zhang R and Xia T: Long non-coding RNA XIST regulates PDCD4 expression by interacting with miR-21-5p and inhibits osteosarcoma cell growth and metastasis. Int J Oncol 51: 1460-1470, 2017

26. Lv GY, Miao J and Zhang XL: Long non-coding RNA XIST promotes osteosarcoma progression by targeting ras-related protein RAP2B via miR-320b. Oncol Res: Apr 12, 2017 (Epub ahead of print)

27. Baker EK, Taylor S, Gupte A, Sharp PP, Walia M, Walsh NC, Zannettino AC, Chalk AM, Burns CJ and Walkley CR: BET inhibitors induce apoptosis through a MYC independent mechanism and synergise with CDK inhibitors to kill osteosarcoma cells. Sci Rep 5: 10120, 2015.

28. Meng Q, Ren C, Wang L, Zhao Y and Wang S: Knockdown of ST6Gal-I inhibits the growth and invasion of osteosarcoma MG-63 cells. Biomed Pharmacother 72: 172-178, 2015.

29. Lin DS, Cai LY, Ding J and Gao WY: Correlation between E-cadherin-regulated cell adhesion and human osteosarcoma MG-63 cell anoikis. Asian Pac J Cancer Prev 15: 8203-8207, 2014.

This work is licensed under a Creative Commons Attribution-NonCommercial-NoDerivatives 4.0 International (CC BY-NC-ND 4.0) License. 